\title{
Shelf Life of Bioactive Compounds from Acerola Pulp (Malpighia spp.) through Freeze-Drying and Microencapsulation
}

\author{
Bruno H. F. Saqueti, ${ }^{a}$ Eloize S. Alves, ${ }^{a}$ Matheus C. Castro, ${ }^{\circ}$ Patrícia D. S. dos Santos, ${ }^{b}$ \\ Nayane B. M. Sinosaki, ${ }^{b}$ Carlos E. R. Senes, ${ }^{\circledR b}$ Jesuí V. Visentainer ${ }^{\circledR a, b}$ and \\ Oscar O. Santos ${ }^{\circledR} *, a, b$ \\ ${ }^{a}$ Departamento de Ciência de Alimentos, Universidade Estadual de Maringá (UEM), \\ 87020-900 Maringá-PR, Brazil \\ ${ }^{b}$ Departamento de Química, Universidade Estadual de Maringá (UEM), \\ 87020-900 Maringá-PR, Brazil
}

\begin{abstract}
Acerola is a tropical fruit with a large industrial and commercial appeal due to its organoleptic qualities and bioactive compound-rich nature. Thus, as bioactive compounds (BC) stability is of great interest in the food industry, assessment of preserving techniques to prolong shelf life has become imperative. Hence, the purpose of this study was to evaluate how storage affects BC conservation in microencapsulated and lyophilized acerola pulps over 180 days. For this matter, antioxidant tests, physical-chemical analyzes, and vitamin $\mathrm{C}$ analysis by mass spectrometry were performed. Results indicated that microencapsulation better preserved the antioxidants in acerola pulp compared to the other preservation technologies employed. This is due to the greater $\mathrm{BC}$ concentration in the microencapsulated, as well as the $\mathrm{pH}$ reduction. Furthermore, over 180 days, microencapsulation better preserved the target compounds in acerola pulp compared to lyophilization, as evidenced by the superior content of $\mathrm{BC}$ in the former treatment, making it an appealing option for the food sector.
\end{abstract}

Keywords: antioxidants, phenolic compounds, anthocyanins, flavonoids, bioactive compounds

\section{Introduction}

Acerola (Malpighia spp.) is a tropical fruit, native to Central America, remarkably rich in bioactive compounds (BC) such as ascorbic acid (AA), phenolic compounds, flavonoids, and anthocyanins. ${ }^{1,2}$ The presence of $\mathrm{BC}$ has often been studied due to its health benefits since research has shown antioxidant, antimutagenic, antidiabetic, antitumor, and hepatoprotective activity attributed to such compounds. ${ }^{3-6}$

Several products are obtained from acerola pulp, such as juices, nectars, and jams. ${ }^{7,8}$ The process to obtain frozen fruit pulp consists of pulping, which removes the insides of the fruit, followed by freezing to preserve nutritional benefits. ${ }^{3,9}$ This procedure greatly decreases losses due to perishability, whilst offering fruits with low seasonality. ${ }^{10}$

Previous studies ${ }^{11-13}$ have demonstrated that the stability of $\mathrm{BC}$ in acerola pulps are affected by $\mathrm{pH}$, light exposure, oxygen, and temperature. Among the main BCs is the AA, a water-soluble and thermolabile vitamin characterized

*e-mail: oliveirasantos.oscardeoliveira@gmail.com as an unstable, easily oxidizable antioxidant, whose bonds can be broken by oxygen, alkaline substances, and high temperatures. ${ }^{14}$ In this sense, fruit pulp preservation methods are being investigated to ensure the bioavailability of nutrients and prevent their loss due to freezing and/or other treatments. ${ }^{15-19}$

Microencapsulation in extracts and/or fruits such as blackberries, blueberries, cherries, jabuticabas, and acerolas has shown positive effects in preserving BC, according to the literature. ${ }^{11,20}$ Spray drying can be used to microencapsulate chemically sensitive compounds such as AA, allowing to obtain dehydrated juices, concentrating the typical active substances, and protecting them in a polymer matrix against oxidation. ${ }^{18}$ Hence, it is critical to evaluate the stability of BCs in acerola pulps submitted to different treatments.

Microencapsulation is a technique that utilizes an encapsulating agent to develop solid, liquid, or volatile particles in a matrix to augment the stability of BCs by providing protection. ${ }^{18,19}$ Important characteristics are attributed to encapsulating agents such as biocompatibility, biodegradability, non-toxicity, and low-cost. ${ }^{20}$ 
Maltodextrin is the most commonly used encapsulating agent that presents these characteristics. It consists of a polysaccharide generated by partial hydrolysis of starch by enzymatic or acid action, giving rise to maltoses and dextrin units allowing it to be widely applied in microencapsulation techniques. ${ }^{21}$

Drying procedures such as freeze-drying are related to the microencapsulation technology, which encourages water remotion from frozen mixtures via sublimation under vacuum pressure utilizing low temperatures followed by desorption. ${ }^{22}$ Freeze-drying ensures higher quality than other techniques, by maintaining the bio-functionality of the BCs whilst prolonging their shelf life without affecting the sensory properties. ${ }^{20}$ Thus, associating microencapsulation with freeze-drying could greatly increase the stability of BCs.

This works aims to develop a chemically stable powdered acerola pulp that can be transported and stored, as well as new approaches to industrialize and market this new product. Based on physical-chemical analyzes, antioxidant assays, and mass spectrometry with electrospray ionization, the effect of storage on the preservation of BC in acerola pulp was studied by microencapsulation and freeze-drying.

\section{Experimental}

Chemicals, reagents, and encapsulating agents

2,2-Diphenyl-1-picrylhydrazyl (DPPH), 6-hydroxy2,5,7,8-tetramethylchroman-2-carboxylic acid (Trolox), gallic acid, Folin-Ciocalteu phenol reagent, quercetin, and maltodextrin (encapsulating agent) were obtained from Sigma-Aldrich (Darmstadt, Germany). Methanol p.a., ethanol p.a., hydrochloric acid, formic acid, and buffer solution (pH 4 and pH 10) were acquired from Synth (São Paulo, Brazil). Potassium chloride, sodium acetate, sodium carbonate, and aluminum chloride were purchased from Dinâmica (São Paulo, Brazil). Ultrapure water was obtained from a Milli-Q ${ }^{\circledR}$ purification system (Millipore, Bedford, United States). All chemicals were analytical grade or high-performance liquid chromatography (HPLC) grade.

\section{Sample preparation and treatments}

Approximately eight kilograms of acerola (Malpighia spp.) were purchased in local commerce in Maringá ( $\left.23^{\circ} 25^{\prime} \mathrm{S}, 51^{\circ} 57^{\prime} \mathrm{W}\right)$, Paraná, Brazil, gathered at the optimum ripening stage (mature) in November 2019. The samples were subjected to steps of hygiene, weighing, and pulping in a fruit pulper (APITEC, DF-100, Maringá, Brazil), carried out in the Vegetable Processing Laboratory of the State University of Maringá. They were submitted to three treatments obtaining the following samples: frozen (FAP), lyophilized (LAP), and microencapsulated and lyophilized (MLAP). The MLAP sample was microencapsulated utilizing a cavitation system in an ultrasonic bath (ELMA, Elmasonic P, São Paulo, Brazil) for $20 \mathrm{~min}$ at $25^{\circ} \mathrm{C}$ and $80 \mathrm{kHz}$, followed by a mechanical stirring on a propeller stirrer (Gehaka AE-40, São Paulo, Brazil) for $20 \mathrm{~min}$ at $25^{\circ} \mathrm{C}$, adding $10 \%(\mathrm{~m} / \mathrm{v})$ maltodextrin as an encapsulating agent to the pulp. ${ }^{2} \mathrm{~A}$ bench freeze dryer (Liotop, L-101, São Carlos, Brazil) was employed for the drying of the LAP and MLAP samples. The treated acerola pulps were vacuum-packed in polyethylene bags and kept in the freezer $\left(-18^{\circ} \mathrm{C}\right)$ until the analyzes were completed.

\section{Preparation of the extracts}

The FAP treatment was thawed at room temperature $\left(25^{\circ} \mathrm{C}\right)$, whereas the LAP and MLAP treatments were resuspended in distilled water utilizing the freezedrying yield. The extracts were prepared according to Rezende et al., ${ }^{11}$ under conditions optimized for the extraction of BC. A $46.5 \%$ ethanol solution acidified with hydrochloric acid $(\mathrm{HCl})$ at $\mathrm{pH} 2$ was used as the extractor solvent, the solvent/pulp ratio was $8.66 \mathrm{~mL} \mathrm{~g}^{-1}$. The extraction was performed in an ultrasonic bath (ELMA, Elmasonic P, São Paulo, Brazil) working at $80 \mathrm{kHz}$ of frequency and $30{ }^{\circ} \mathrm{C}$ of temperature for $50 \mathrm{~min}$. The resulting extract was filtered through Whatman No. 3 filter paper, and the liquid was then concentrated at $55^{\circ} \mathrm{C}$ on a rotary evaporator (Fisatom 802, São Paulo, Brazil) until around $95 \%$ of the extraction solvent was removed, then the concentrated extract was resuspended in acidified water at $\mathrm{pH}$ 2. The extracts were stocked in an amber flask and stored in a freezer $\left(-18{ }^{\circ} \mathrm{C}\right)$ for further analysis.

\section{DPPH assay}

The DPPH radical scavenging assay was performed according to the method described by Brand-Williams et al..$^{23}$ with modifications. ${ }^{24}$ The wavelength $(\lambda)$ of $517 \mathrm{~nm}$ was used by a spectrophotometer (Genesys $10-\mathrm{S}$ UV/Vis, Rochester, USA). A standard curve using Trolox (0-0.3 $\left.\mathrm{mg} \mathrm{mL}^{-1}\right)$ was used to calculate antioxidant activity, and the result was represented as $\mu$ mol of trolox equivalent (TE) $\mathrm{g}^{-1}$ of sample. The DPPH assay was carried out every 15 days for 180 days after obtaining the treatments.

$\mathrm{pH}$ and soluble solids analysis

A pH meter (Digimed DM-22, São Paulo, Brazil) was 
used to measure the $\mathrm{pH}$ values at room temperature, and the refractive index (soluble solids) was measured in a refractometer (Abbe RTA-100, São Paulo, Brazil) and expressed in ${ }^{\circ}$ Brix. ${ }^{25}$ The evaluation was carried out at the storage times of 0,90 , and 180 days.

\section{Colorimetric analysis}

Color analysis was performed using a digital colorimeter (Konica Minolta CR-400, Osaka, Japan), obtaining the values of $\mathrm{L}^{*}$ (lightness), chromaticity a* (intensity of $\mathrm{red} /$ green), and $\mathrm{b}^{*}$ (intensity of yellow/blue) according to the model of the International Commission on Illumination (CIE). ${ }^{26}$ The evaluation was carried out at storage times of 0,90 , and 180 days.

\section{Total phenolic compounds (TPC)}

The TPC content was determined using the FolinCiocalteu reagent according to the method described by Singleton and Rossi. ${ }^{27}$ The TPC content was calculated using a standard curve prepared from aqueous solutions of gallic acid (0.1-1 $\left.\mathrm{mg} \mathrm{mL}^{-1}\right)$ and the result was expressed in $\mathrm{mg}$ of gallic acid equivalent (GAE) per $100 \mathrm{~g}$ of sample. The evaluation was carried out at storage times of 0,90 , and 180 days.

\section{Total flavonoids (TF)}

The TF content of acerola pulps submitted to different treatments was determined at three storage periods $(0$, 90, and 180 days) following the method described by Moo-Huchin et al. ${ }^{28}$ The absorbance was measured at $415 \mathrm{~nm}$ using a spectrophotometer (Genesys 10-S UV/Vis, Rochester, USA). The content was calculated using an analytical curve of standard quercetin (0.05-0.5 $\left.\mathrm{mg} \mathrm{mL}^{-1}\right)$. Results were expressed in $\mathrm{mg}$ of quercetin equivalent $(\mathrm{QE})$ per $100 \mathrm{~g}$ of sample.

Total anthocyanins (TA)

TA content determination was carried out following the $\mathrm{pH}$ difference method. ${ }^{29}$ The concentration of monomeric pigments in the extract was expressed as cyanidin3 -glycoside mg $100 \mathrm{~g}^{-1}$. The analysis was performed at three storage periods (0, 90, and 180 days).

AA identification by direct infusion electrospray ionization mass spectrometry (ESI-MS)

A XevoTQD ${ }^{\mathrm{TM}}$ triple quadrupole mass spectrometer
(Waters, Massachusetts, United States) equipped with a $\mathrm{Z}$ spray ${ }^{\mathrm{TM}}$ electrospray ionization source operating in negative mode was utilized to identify AA in treated extracts of acerola stored for 0,90 , and 180 days. Mass spectra were acquired in scanning mode using $\mathrm{m} / \mathrm{z}$, ranging from 100 to 700. Before infusion into the spectrometer, $50 \mu \mathrm{L}$ of the extract was dissolved in $950 \mu \mathrm{L}$ of ultrapure water, then $1 \mathrm{~mL}$ of this solution was transferred to a vial and $20 \mu \mathrm{L}$ of $1 \%$ formic acid solution was added. The sample was infused at a flow rate of $10 \mu \mathrm{L} \mathrm{min}{ }^{-1}$. The working conditions of the ionization source were as follows: capillary voltage of $3.06 \mathrm{kV}$, source temperature of $150{ }^{\circ} \mathrm{C}$, cone flow of $40 \mathrm{~L} \mathrm{~h}^{-1}$, gas flow (nitrogen) for desolvation of $400 \mathrm{~L} \mathrm{~h}^{-1}$, desolvation temperature of $350^{\circ} \mathrm{C}$, and the gas flow cone of $30 \mathrm{~L} \mathrm{~h}^{-1}$. Data were acquired and processed using MassLynx v 4.1 software and AA was identified by comparison with the assistance of a database. Calibration was carried out with a standard solution of phosphoric acid utilizing the center of mass in the first and second quadrupoles, as established by the company protocol (XevoAcquity ${ }^{\mathrm{TM}}$, Waters, Milford, MA, USA).

\section{Statistical analysis}

Analyzes were performed in triplicate and results were expressed as mean \pm standard deviation. Results were evaluated by analysis of variance (ANOVA) using the Tukey's test with a significance level of 5\% $(\alpha=0.05)$ using Assistat 7.7 software. ${ }^{30}$

\section{Results and Discussion}

\section{DPPH assay}

Figure 1 shows the concentration data from the DPPH assay, which were assessed every two weeks for 180 days.

The highest concentrations in DPPH assay at time zero were achieved in LAP and MLAP treatments, with no significant difference between both $(p>0.05)$. The data obtained corroborate those presented by Rezende et al., ${ }^{11}$ who found similar values in the optimization of an ultrasound-assisted extraction of acerola residue, with the highest value being $181.78 \mu \mathrm{M} \mathrm{TE} \mathrm{g}^{-1}$. After 150 days, the values decreased significantly and remained stable until 180 days. The MLAP sample obtained the highest final concentration. The antioxidant activity is influenced by the concentration of BCs in the sample, i.e., if the sample is photochemically degraded, the antioxidant activity will be affected. The decrease in antioxidant activity in fruits during storage was previously verified in the work of Michalczyk et al., ${ }^{31}$ who, despite employing the freeze- 


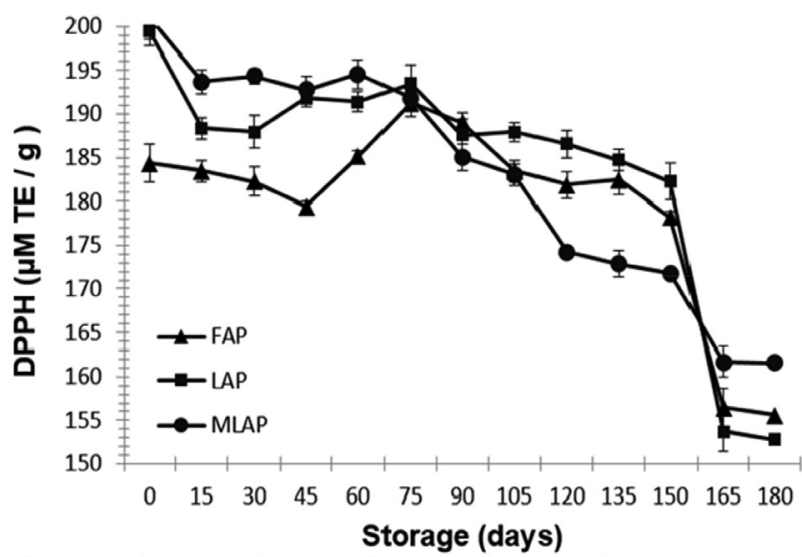

Figure 1. DPPH (radical scavenging activity) assay carried out every 15 days of storage. FAP: frozen acerola pulp; LAP: lyophilized acerola pulp; MLAP: microencapsulated and lyophilized acerola pulp. Values are expressed as mean \pm triplicate standard deviation.

drying treatment and spiking samples with high content of polyphenols and anthocyanins, reported a decrease in antioxidant activity.

$\mathrm{pH}$ and soluble solids analysis

The data from the analysis of $\mathrm{pH}$ and soluble solids are exhibited in Figure 2.

The $\mathrm{pH}$ (Figure 2a) results showed changes depending on the treatment. Freeze-dried samples LAP and MLAP achieved lower values, indicating a significant difference $(p \leq 0.05)$. Storage time resulted in changes in $\mathrm{pH}$, and the FAP and LAP samples showed a significant reduction in the two time periods tested, indicating that the compounds in these samples were stable. The statistical insignificance between 90 and 180 days for MLAP demonstrates that the microencapsulation successfully enhanced the stability of the sample. When analyzed in vitro, $\mathrm{pH}$ levels affect the antioxidant properties of phenolic compounds, which are more sensitive to changes in $\mathrm{pH}$ than the numbers and positions of surrogate groups. In this case, high $\mathrm{pH}$ values resulted in high antioxidant activity, whereas low $\mathrm{pH}$ values resulted in low antioxidant activity. ${ }^{29}$

Soluble solids (Figure $2 \mathrm{~b}$ ) are total solids dissolved in water comprising sugars, organic acids, soluble proteins, and salts, which are counted and measured as the sum of those solids and given in ${ }^{\circ} \mathrm{Brix}$. In the time of zero-days, the values of $5.97 \pm 0.04$ and $6.13 \pm 0.04{ }^{\circ}$ Brix in FAP and LAP samples, respectively, did not differ substantially $(p>0.05)$, and with the passage of storage time, an increase in the content of soluble solids with proportions were observed. The MLAP sample had a value of $10.93 \pm 0.49{ }^{\circ}$ Brix at zero-days, which was greater than the other treatments, justifying the use of an encapsulating agent in the sample. The MLAP sample
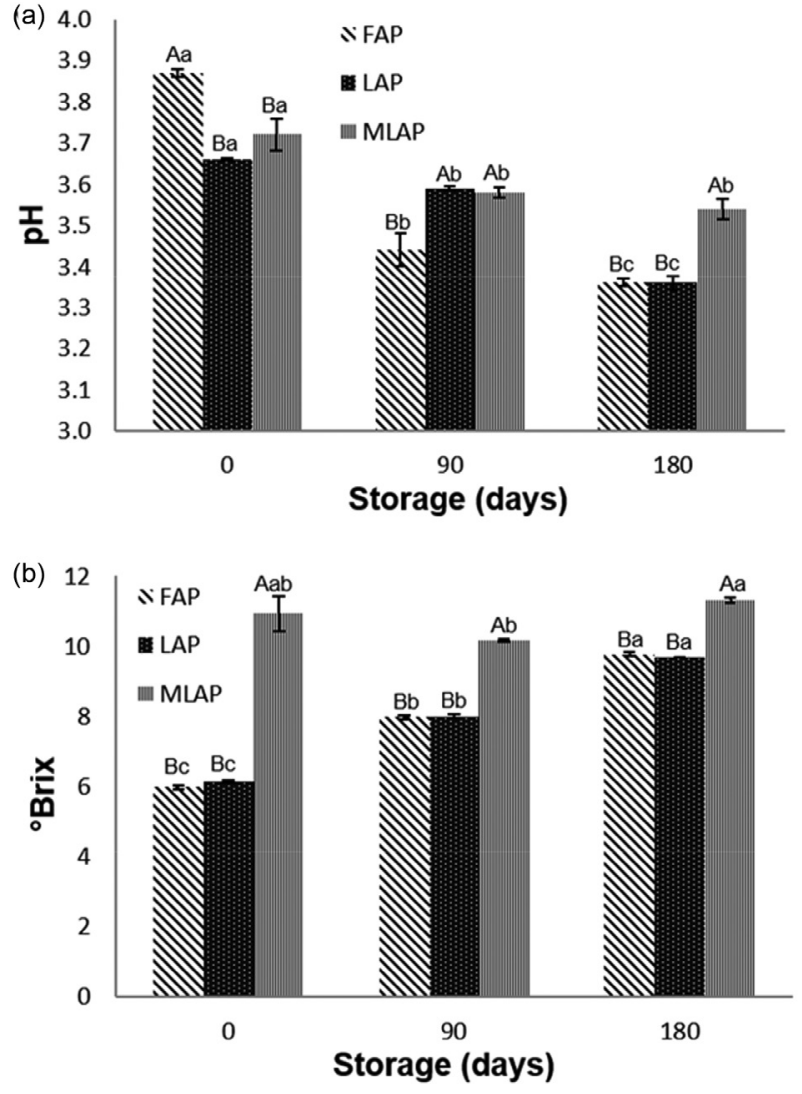

Figure 2. (a) $\mathrm{pH}$ (hydrogen potential) analysis; (b) soluble solids analysis. FAP: frozen acerola pulp; LAP: lyophilized acerola pulp; MLAP: microencapsulated and lyophilized acerola pulp, in storage times of 0,90 , and 180 days in different treatments. Data are expressed as mean \pm triplicate standard deviation. Equal capital letters mean that the treatments do not differ significantly by the Tukey's test at $5 \%$ probability, equal lower letters mean that the storage times (days) do not differ significantly by the Tukey's test at $5 \%$ probability.

behaved differently over the storage period, with a modest decrease in insoluble solids after 90 days and an increase after 180 days, indicating a significant difference $(p \leq 0.05)$. Jeong et al. ${ }^{32}$ reported a gradual increase in soluble solids in stored chilled kiwi cultivars due to cell wall degradation, which makes organic acids more accessible. The values of the soluble solids found in FAP and LAP are close to the values obtained by Souza et al.,$^{33}$ which obtained values ranging from 6.8 to $8.2{ }^{\circ} \mathrm{Brix}$ in immersion treatment of acerola.

\section{Colorimetric analysis}

Color is an important attribute for food quality and consumer acceptance. The coloration observed in fruits such as ripe acerola is primarily determined by the presence of natural pigments, which may be influenced by several factors including fruit maturation stage, type of processing, oxidation reactions, among others. ${ }^{34}$ Color is related to changes in $\mathrm{pH}$, which in turn is related to BCs content, thus 
colorimetric analysis also provides relevant information regarding the chemical behavior of samples.

The results of the colorimetric analysis are presented in Figure 3 based on three parameters ( $\mathrm{L}^{*}, \mathrm{a}^{*}$, and $\mathrm{b}^{*}$ ), taking into account the angle at which the sample is detected, which may affect the reflectance curve, used as a reference

(a)
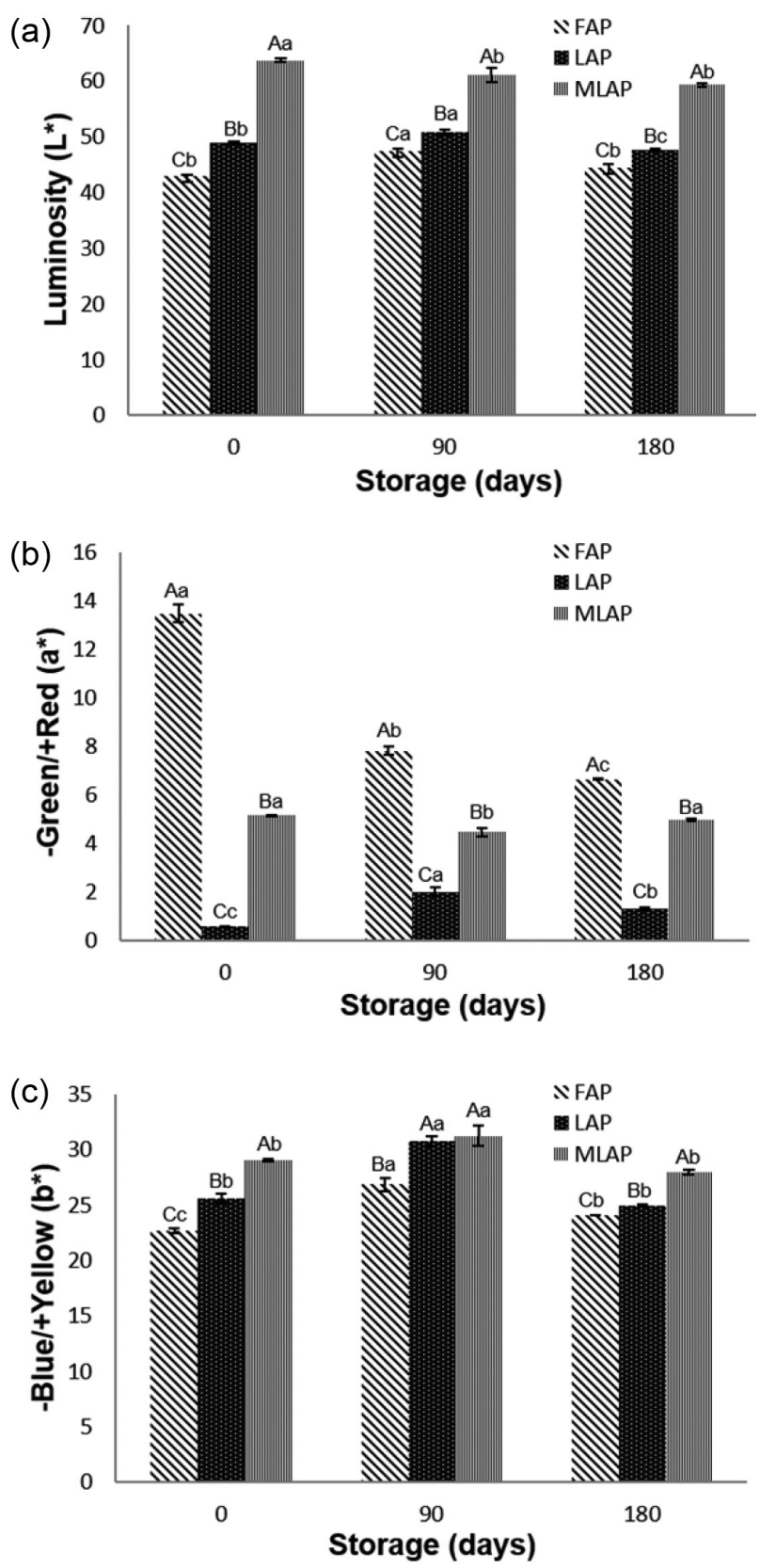

Figure 3. Colorimetric analysis (a) $L^{*}$ brightness; (b) a* intensity of red when the value is positive/green when the value is negative; (c) $b^{*}$ yellow intensity when the value is positive/blue when the value is negative, at the storage times of 0,90 , and 180 days in different treatments. FAP: frozen acerola pulp; LAP: lyophilized acerola pulp; MLAP: microencapsulated and lyophilized acerola pulp. Data are expressed as mean \pm triplicate standard deviation. Equal capital letters mean that treatments do not differ significantly by Tukey's test at $5 \%$ probability, equal lower-case letters mean that storage times do not differ significantly by Tukey's test at $5 \%$ probability. for the CIE (International Commission on Illumination) curve. ${ }^{26}$

Figure 3 a refers to the lightness $\left(\mathrm{L}^{*}\right)$ and/or contrast of the sample, in which at zero-days the values for FAP, LAP, and MLAP differed statistically ( $p \leq 0.05)$ from each other due to the freeze-drying process and maltodextrin addition. Both processes will contribute to an increase in lightness, particularly the addition of maltodextrin, since the encapsulating white powder dissolves in the acerola pulp, generating a clear sample. After 90 and 180 days of storage, the samples retained their values without significant differences $(p>0.05)$.

Figure $3 b$ concerns chromaticity $\left(a^{*}\right)$, i.e., visible light radiation characterized by two chromatic coordinates. Results ranged from 0.56 to 13.49 , with positive values and their color tending towards the red color spectrum. The sample with the strongest red trend at zero-day storage time was FAP, followed by MLAP and LAP, with a significant difference $(p \leq 0.05)$ between them. This factor could have been generated by the $\mathrm{pH}$ impacting the balance between the different forms of anthocyanins and, as a result, in the modification of pigments that exhibit red coloring. Concerning 90 days storage period, the FAP, and MLAP values were reduced, while the LAP values were increased in intensity, differing significantly ( $p \leq 0.05)$. At 180 days FAP and LAP showed a small reduction and MLAP remained stable without significant differences $(p>0.05)$.

Regarding $b^{*}$ (Figure 3c), it refers to the blue/yellow chromatic intensity, and the samples showed values ranging from 22.64 and 31.23, with positive values and their color tending towards the yellow color spectrum. The $b^{*}$ values for the different storage times were not significantly different $(p>0.05)$. The trend toward red and yellow is expected due to the presence of natural pigments such as anthocyanins and carotenoids found in the samples.

\section{Bioactive compounds}

Figure 4 shows the results concerning the BC study. For TPC (Figure 4a), the results demonstrate a statistically significant difference in $\mathrm{BC}$ content at zero-days among the treatments, with MLAP exhibiting the highest concentration, followed by LAP and FAP.

After 90 days of storage, an increase and decrease in TPC was observed for FAP (481.84 $\pm 0.65 \mathrm{mg}$ GAE per $100 \mathrm{~g})$ and LAP (455.17 $\pm 8.74 \mathrm{mg} \mathrm{GAE} \mathrm{per} 100 \mathrm{~g})$, respectively, while TPC in MLAP (493.16 $\pm 3.31 \mathrm{mg} \mathrm{GAE}$ per $100 \mathrm{~g}$ ) slightly varied. Regarding 180 days of storage, TPC content for samples FAP $(501.78 \pm 0.31 \mathrm{mg}$ GAE per $100 \mathrm{~g})$ and MLAP (535.56 $\pm 0.56 \mathrm{mg}$ GAE per 

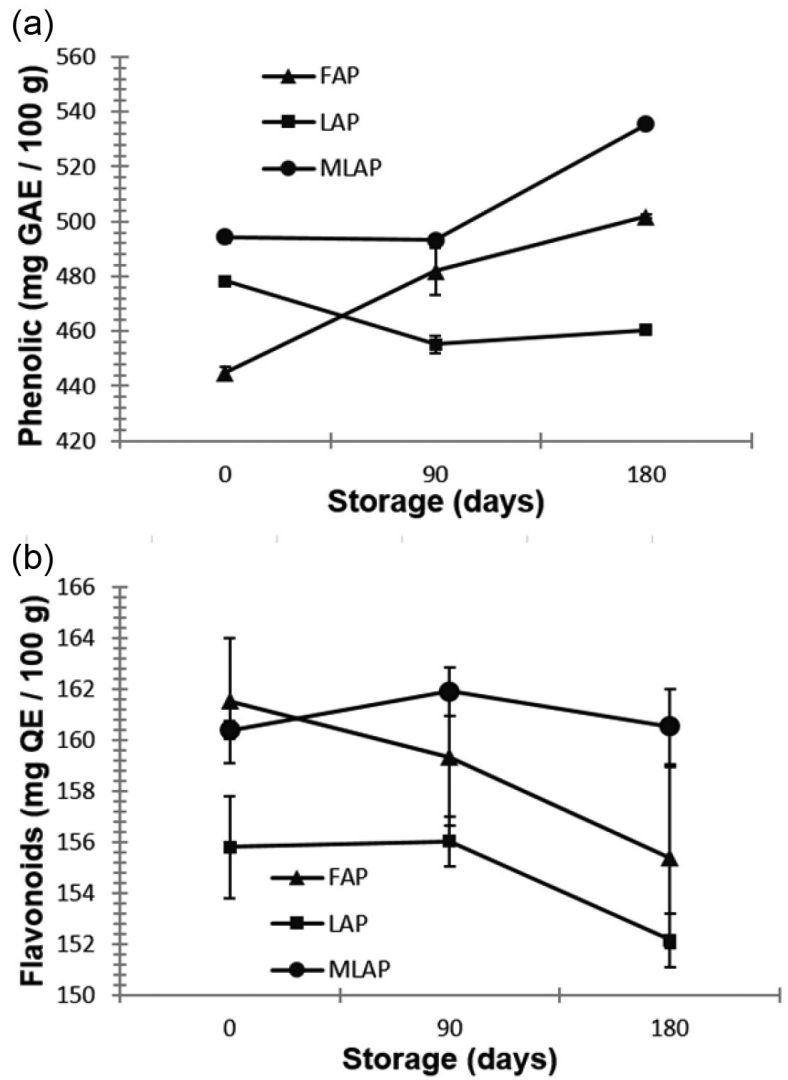

(c)

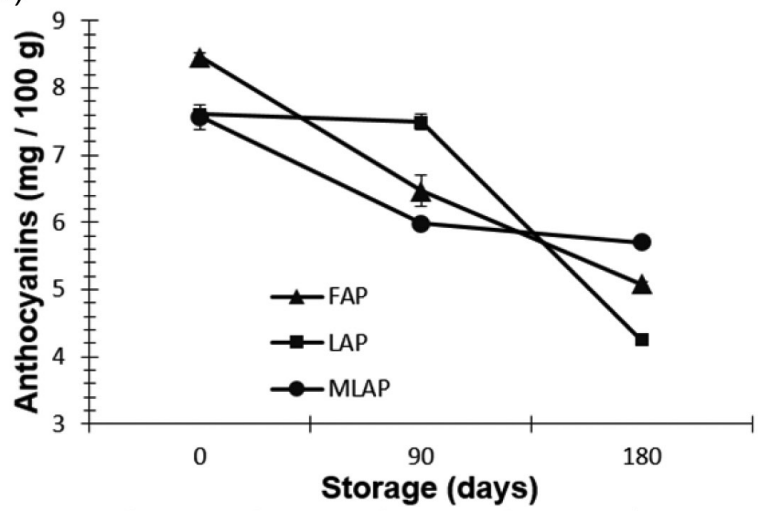

Figure 4. Bioactive compounds content. (a) Total phenolic compounds (TPC); (b) total flavonoids (TF); (c) total anthocyanins (TA) at storage times of 0,90 , and 180 days in different treatments. FAP: frozen acerola pulp; LAP: lyophilized acerola pulp; MLAP: microencapsulated and lyophilized acerola pulp. Data are expressed as mean \pm triplicate standard deviation.

$100 \mathrm{~g})$ substantially increased whereas for LAP $(460.34 \pm 0.57 \mathrm{mg}$ GAE per $100 \mathrm{~g})$ no significant alteration was verified. The increase in TPC concentration during storage was observed by Mallik and Hamilton ${ }^{35}$ in a study evaluating wild blueberries, corroborating the results obtained in this work.

The increase in TPC during storage at freezing temperatures is due to the formation of secondary metabolites, originated from the breakdown of cellular components of acerola pulps and hydrolysis of glycosides. Another explanation could be the ripening stage of the fruits, since less mature fruits could continue the biosynthesis of phenolics even at low temperatures. ${ }^{36}$ The high level of phenolic compounds detected in acerola pulp are attributed to classes of compounds derived from benzoic acid (gallic acid and syringic acid), phenylpropanoid derivatives ( $p$-cumaric, ferulic, caffeic, and chlorogenic acids), flavonoids, and anthocyanins. ${ }^{37}$

The main flavonoids commonly found in acerola are catechin, epicatechin, epigallocatechin gallate, quercetin, rutin, kaempferol, luteolin, dihydroquercetin, procyanidin B1, and aceronidin. ${ }^{37}$ Figure $4 \mathrm{~b}$ demonstrates that prior to storage (zero-days) TF concentration of FAP and MLAP were statistically equal, and superior to that of LAP.

The samples remained stable for 90 and 180 days of storage with no significant differences $(p>0.05)$ over the storage time. Over the 180-days storage period, MLAP exhibited the greatest concentration of TF, hence indicating that microencapsulation successfully helped to preserve the flavonoids of samples, concurring with the findings reported by Sansone et al. ${ }^{38}$ which stated the positive effects of microencapsulation on shelf life and compound stability.

For TA (Figure 4c), in the storage time of zero-days, the FAP showed a higher concentration, differing statistically ( $p \leq 0.05$ ) from LAP and MLAP, that is, freeze-drying and microencapsulation affected the initial TA concentrations in the samples. After 90 days of storage, TA concentrations in the FAP and MLAP treatments were reduced by 23.5 and $21 \%$, respectively, whereas LAP remained stable with no significant alteration $(p>0.05)$. Past 180 days of storage, the samples with lower concentrations of TA were FAP and LAP, decreasing 21.4 and $43.2 \%$ over 90 days, respectively, while MLAP remained stable, consequently becoming the treatment with the highest concentration of AT over the 180-days storage period. According to Mahdavi et al. ${ }^{39}$ anthocyanins are hydrophilic dyes specifically compatible with a water-based gel formulation, such as maltodextrin. After drying, the production of spherical particles is observed, coating the pigment in the peripheral region of the microcapsules.

AA identification by mass spectrometry with direct electrospray infusion (ESI-MS)

To define the best method for preserving the BCs of acerola pulps, ESI-MS analysis was performed to verify the storage stability of AA in FAP, LAP, and MLAP over 180 days, and the results are illustrated in Figure 5. 
As shown by Figure 5, a slight variation in AA content was observed for the three different treatments throughout the first 90 days of storage, followed by a more substantial change in the last 90 days of storage, especially for MLAP and LAP. Over 90 days of storage the AA content of all three samples increased, with the highest increase being detected for FAP (39.2\%), followed by MLAP (20.3\%) and LAP $(8.1 \%)$, respectively. After 180 days of storage a drastic increase in AA concentration was verified for LAP (39.9\%) and MLAP (60.6\%) whereas only a slight decrease in the compound concentration was observed for FAP.

This effect is due to the intrinsic characteristics of AA, mainly because of its high-water solubility, which directly affects oxidation and reduction reactions. For FAP, after 90 days, its concentration decreased, probably due to the interaction of the AA molecules with water, since hydrogen bonds reduce the movement of molecules, mainly in solid materials. Moreover, the size and concentrations of the particles directly affect the oxidation reactions, thus reducing the concentration. As previously said, AA concentration in LAP and MLAP increased over the days since the water was removed during the freeze-drying process. As a result, the encapsulated component is released, resulting in dehydroascorbic acid as a decomposition product from an incomplete AA degradation. ${ }^{40}$

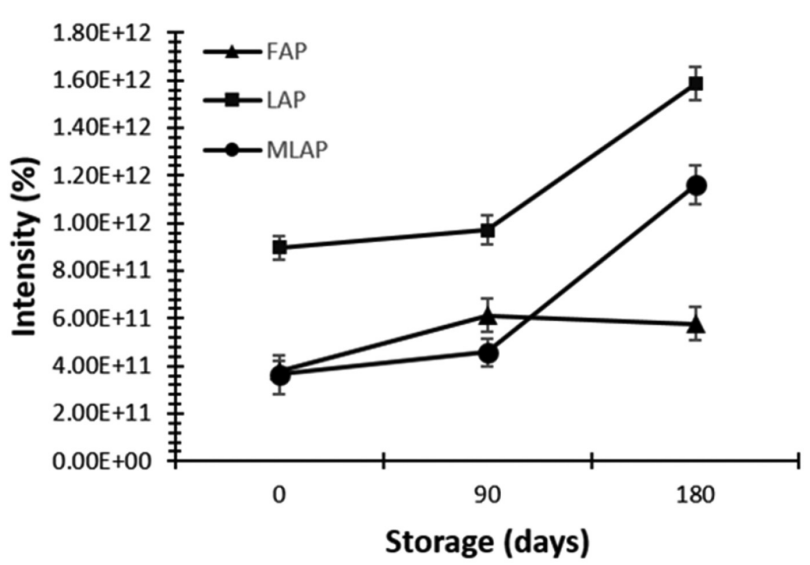

Figure 5. The intensity of ascorbic acid (AA) in acerola in the storage time of 0,90 , and 180 days in different treatments. FAP: frozen acerola pulp; LAP: lyophilized acerola pulp; MLAP: microencapsulated and lyophilized acerola pulp.

However, it is important to note that AA stability is influenced by the intrinsic properties of the product and by the characteristics of the process that are subjected to different handling and storage conditions. These conditions are influenced by aerobic or anaerobic pathways, as well as prolonged storage, $\mathrm{pH}$, humidity, temperature, cold injury, water, the acidity of the medium, the presence of metallic ions, concentration of enzymes, proteins, carbohydrates and amino acids contained in the sample. ${ }^{41}$

\section{Conclusions}

Association of microencapsulation and lyophilization was successful in providing an acerola pulp with higher quality and BCs stability. The employed technology successfully aided in preserving bioactive compound concentration and antioxidant activity throughout the 180 -days storage period. Furthermore, the MLAP treatment achieved higher $\mathrm{pH}$ stability over the evaluated storage period. There was a significant increase in AA concentration in LAP and MLAP treatments, i.e., samples that were dried (lyophilization) were able to preserve and concentrate AA. Thus, the present study showed that the microencapsulation technique provides a powder product, which presents stability of bioactive compounds. Moreover, it brings an innovative technique for the food industry, facilitating storage and transportation of the pulp, which can be used alone or in the development of new products.

\section{Supplementary Information}

Supplementary data are available free of charge at http://jbcs.sbq.org.br as PDF file.

\section{Acknowledgments}

The authors thank Conselho Nacional de Desenvolvimento Científico e Tecnológico, Brazil (CNPq), Coordenação de Aperfeiçoamento de Pessoal de Nível Superior (CAPES), and Fundação Araucária de Apoio ao Desenvolvimento Científico e Tecnológico do Paraná for their financial support.

\section{Author Contributions}

Bruno H. F. Saqueti was responsible for methodology, investigation, formal analysis, and writing original draft; Eloize S. Alves for investigation and formal analysis; Matheus C. Castro for investigation and formal analysis; Patrícia D. S. Santos for investigation, formal analysis, and validation; Nayane B. M. Sinosaki for investigation, formal analysis, and validation; Carlos E. R. Senes for writing-review and editing; Jesuí V. Visentainer for funding acquisition and supervision; Oscar O. Santos for conceptualization, visualization and supervision.

\section{References}

1. Silva, P. B.; Duarte, C. R.; Barrozo, M. A. S.; Innovative Food Sci. Emerging Technol. 2019, 52, 350. 
2. Rezende, Y. R. R. S.; Nogueira, J. P.; Narain, N.; Food Chem. 2018, 254, 281.

3. Paz, M.; Gúllon, P.; Barroso, M. F.; Carvalho, A. P.; Domingues, V. F.; Gomes, A. M.; Becker, H.; Longhinotti, E.; DelerueMatos, C.; Food Chem. 2015, 172, 462.

4. Düsman, E.; Almeida, I. V.; Tonin, L. T. D.; Vicentini, V. E. P.; Genet. Mol. Res. 2016, 15, gmr15049036.

5. Barbalho, S. M.; Damasceno, D. C.; Spada, A. P. M. H.; Palhares, M.; Martuchi, K. A.; Oshiiwa, M.; Sazaki, V.; da Silva, V. S.; Exp. Diabetes Res. 2011, ID 173647.

6. Luveena, A. A. M.; Karthiayini, K.; Sreekumar, K. P.; Int. J. Res. Phytochem. Pharmacol. 2012, 2, 45.

7. Bortolotti, C. T.; Santos, K. G.; Francisquetti, M. C. C.; Duarte, C. R.; Barrozo, M. A. S.; Can. J. Chem. Eng. 2013, 91, 1871.

8. Saraji, M.; Boroujeni, M. K.; Anal. Bioanal. Chem. 2014, 406, 2027.

9. da Silva, L. M. R.; de Figueiredo, E. A. T.; Ricardo, N. M. P. S.; Vieira, I. G. P.; Figueiredo, R. W.; Brasil, I. M.; Gomes, C.; Food Chem. 2014, 143, 398.

10. da Costa, G. A.; Mercadante, A. Z.; J. Food Compos. Anal. 2018, 68, 53.

11. Rezende, Y. R. R. S.; Nogueira, J. P.; Narain, N.; LWT - Food Sci. Technol. 2017, 85, 158.

12. de Rosso, V. V.; Mercadante, A. Z.; Food Chem. 2007, 103, 935.

13. Jaeschke, D. P.; Marczak, L. D. F.; Mercali, G. D.; Food Chem. 2016, 199, 128.

14. Manela-Azulay, M.; Lacerda, C. A. M.; Perez, M. A.; Filgueira, A. L.; Cuzzi, T.; An. Bras. Dermatol. 2003, 78, 265.

15. Robert, P.; Torres, V.; García, P.; Vergara, C.; Sáenz, C.; LWT - Food Sci. Technol. 2015, 60, 1039.

16. Santana, A. A.; Cano-Higuita, D. M.; de Oliveira, R. A.; Telis, V. R. N.; Food Chem. 2016, $212,1$.

17. Braga, M. B.; Veggi, P. C.; Codolo, M. C.; Giaconia, M. A.; Rodrigues, C. L.; Braga, A. R. C.; Food Res. Int. 2019, 125, 108557.

18. Sampaio, G. L. A.; Pacheco, S.; Ribeiro, A. P. O.; Galdeano, M. C.; Gomes, F. S.; Tonon, R. V.; LWT 2019, 116, 108589.

19. Pacheco, C.; García-Martínez, E.; Moraga, G.; Piña, J.; Nazareno, M. A.; Martínez-Navarrete, N.; Powder Technol. 2020, 362, 11.

20. Tarone, A. G.; Cazarin, C. B. B.; Marostica Jr., M. R.; Food Res. Int. 2020, 133.

21. Ribeiro, A. M.; Estevinho, B. N.; Rocha, F.; Trends Food Sci. Technol. 2020, 105, 454.

22. Ezhilarasi, P. N.; Karthik, P.; Chhanwal, N.; Anandharamakrishnan, C.; Food Bioprocess Technol. 2013, 6, 628 .
23. Brand-Williams, W.; Cuvelier, M. E.; Berset, C.; LWT - Food Sci. Technol. 1995, 28, 25.

24. Boroski, M.; de Aguiar, A. C.; Boeing, J. S.; Rotta, E. M.; Wibby, C. L.; Bonafé, E. G.; de Souza, N. E.; Visentainer, J. V.; Food Chem. 2011, 125, 696.

25. Association of Official Analytical Chemists (AOAC); Official Methods of Analysis of the Association of Official Analytical Chemists, 18 ${ }^{\text {th }}$ ed.; AOAC: Gaithersburg, 2005.

26. McGuire, R. G.; HortScience 1992, 27, 1254.

27. Singleton, V. L.; Rossi, J. A.; Am. J. Enol. Vitic. 1965, 16, 144.

28. Moo-Huchin, V. M.; Moo-Huchin, M. I.; Estrada-León, R. J.; Cuevas-Glory, L.; Estrada-Mota, I. A.; Ortiz-Vázquez, E.; Betancur-Ancona, D.; Sauri-Duch, E.; Food Chem. 2015, 166, 17.

29. di Majo, D.; la Neve, L.; la Guardia, M.; Casuccio, A.; Giammanco, M.; J. Food Compos. Anal. 2011, 24, 265.

30. Francisco, A. S. S.; Carlos, A. V. A.; Afr. J. Agric. Res. 2016, $11,3733$.

31. Michalczyk, M.; MacUra, R.; Matuszak, I.; J. Food Process. Preserv. 2009, 33, 11.

32. Jeong, H. R.; Cho, H. S.; Cho, Y. S.; Kim, D. O.; Food Sci. Biotechnol. 2020, 29, 1763.

33. Souza, K. O.; Moura, C. F. H.; Lopes, M. M. A.; Rabelo, M. C.; Miranda, M. R. A.; Rev. Bras. Frutic. 2017, 39, e574.

34. de Araújo, F. F.; de Paulo Farias, D.; Neri-Numa, I. A.; Dias-Audibert, F. L.; Delafiori, J.; de Souza, F. G.; Catharino, R. R.; do Sacramento, C. K.; Pastore, G. M.; Food Chem. 2021, 338, 127747.

35. Mallik, A. U.; Hamilton, J.; J. Food Sci. Technol. 2017, 54, 1545 .

36. Celli, G. B.; Ghanem, A.; Brooks, M. S.-L.; Food Rev. Int. 2016, 32, 280.

37. Belwal, T.; Devkota, H. P.; Hassan, H. A.; Ahluwalia, S.; Ramadan, M. F.; Mocan, A.; Atanasov, A. G.; Trends Food Sci. Technol. 2018, 74, 99.

38. Sansone, F.; Picerno, P.; Mencherini, T.; Villecco, F.; D’Ursi, A. M.; Aquino, R. P.; Lauro, M. R.; J. Food Eng. 2011, 103, 18.

39. Mahdavi, S. A.; Jafari, S. M.; Assadpoor, E.; Dehnad, D.; Int. J. Biol. Macromol. 2016, 85, 379.

40. Yuan, J. P.; Chen, F.; J. Agric. Food Chem. 1998, 46, 5078.

41. Mercali, G. D.; Gurak, P. D.; Schmitz, F.; Marczak, L. D. F.; Food Chem. 2015, 171, 200.

Submitted: June 2, 2021

Published online: July 5, 2021 